\title{
DIVERSIDAD DE LA ENTOMOFAUNA ACUÁTICA Y CALIDAD DE AGUA EN QUEBRADAS DEL RÍO SAN JUAN, CHOCÓ - COLOMBIA
}

\section{DIVERSITY OF AQUATIC ENTOMOFAUNA AND WATER QUALITY IN STREAMS OF SAN JUAN RIVER, CHOCÓ - COLOMBIA}

\author{
Zuleyma Mosquera Murillo ${ }^{1}$, Mayrin Minerva Mosquera Mosquera $^{2}$ \\ ${ }^{1}$ Bióloga, M.Sc. Biología, Profesora Asistente, Programa de Biología. Universidad Tecnológica del Chocó, Ciudadela Uni- \\ versitaria, B/ Nicolás Medrano, Quibdó - Chocó, Colombia, e-mail: d-zuleyma.mosquera@utch.edu.co; ${ }^{2}$ Bióloga, Grupo de \\ Limnología. Universidad Tecnológica del Chocó, Ciudadela Universitaria, B/ Nicolás Medrano, Quibdó - Chocó, Colombia, \\ e-mail: mairynmosquera@hotmail.com
}

Rev. U.D.C.A Act. \& Div. Cient. 20(1): 149-161, Enero-Junio,2017

\section{RESUMEN}

A pesar de su destacada importancia, los ecosistemas acuáticos de la región del Chocó vienen sufriendo grandes impactos, causados por las actividades humanas, entre ellas, la minería, por ser esta unas de las principales actividades económicas de la región. En este sentido y considerando el valor de los insectos acuáticos, como bioindicadores de calidad de agua, el objetivo de este estudio fue evaluar la diversidad de la entomofauna acuática y la calidad de agua, en quebradas de la cuenca del río San Juan (Chocó-Colombia). Con tal propósito, se realizaron colectas de insectos acuáticos, entre mayo y agosto de 2015, en múltiples hábitats (piedras, hojarasca, vegetación ribereña), de cinco quebradas de la cuenca estudiada. Asimismo, se midieron algunas variables fisicoquímicas del agua y se evaluaron los índices BMWP/Col y ASPT, para determinar la calidad del agua. Un total de 4.100 individuos fueron colectados, correspondientes a nueve órdenes, 37 familias y 59 géneros. Ephemeroptera fue el más abundante de los órdenes $(34,32 \%)$ y el de mayor riqueza taxonómica (13 géneros). En relación a la diversidad, casi todas las quebradas estudiadas corresponden a diversidad alta, a excepción de Guapandó, con diversidad media, pero no se presentaron diferencias significativas $(p>0,05)$, mientras que Raspadurita fue la que presentó la mayor riqueza taxonómica, con el $76,56 \%$ de los géneros registrados en el estudio. Los valores de similaridad indican que las quebradas muestran una alta variabilidad en sus comunidades. Los valores del índice BMWP/Col clasifican a las quebradas como muy limpias, con calidad buena, mientras que el índice ASPT, las clasifica como ligeramente contaminadas, con calidad aceptable.

\section{SUMMARY}

Despite its outstanding importance, the aquatic ecosystems of the Chocó region are suffering great impacts caused by human activities, including mining; being the one of the main economic activities of the region. In this sense and considering the value of aquatic insects as bio indicators of water quality, the objective of this study was to evaluate the diversity of aquatic entomofauna and water quality in streams of the San Juan River (Chocó-Colombia). For this purpose, aquatic insects were collected between May and August 2015 in multiple habitats (stones, leaf litter and riparian vegetation) of five streams of the studied basin. In addition, some physicochemical variables of water were measured and BMWP/Col and ASPT indices were evaluated for water quality. 4119 individuals were collected, corresponding to nine orders, 37 families and 59 genera. Ephemeroptera was the most abundant of the orders (34.32\%), and the highest taxonomic richness (13 genera). With regard to diversity, almost all the streams studied correspond to high diversity, except Guapandó, but no significant differences $(p>0.05)$ were presented and Raspadurita was the one that presented the greatest taxonomic richness with $76.56 \%$ of the genera recorded in the study. Similarity values indicate that the streams exhibit high variability in their communities. BMWP/Col index values classified streams as very clean with good quality, while the ASPT index classifies as slightly contaminated with acceptable quality.

Key words: Aquatic insects, bioindication, water sources.

Palabras clave: Bioindicación, fuentes hídricas, insectos acuáticos. 


\section{INTRODUCCIÓN}

Los sistemas lóticos son considerados uno de los recursos naturales más importantes para la vida; sin embargo, en las últimas décadas han sufrido grandes impactos, causados por las actividades humanas, que han llevado a una reducción sustancial de la diversidad acuática, gran parte, de la cual, está representada por los insectos acuáticos, que son, con frecuencia, el principal componente animal de estos ecosistemas (Lara-Lara et al. 2008).

Esta problemática ha generado un creciente interés por conocer el estado de salud de los ecosistemas acuáticos y su evolución en el tiempo, con el fin de encontrar estándares de juicio de "Calidad de agua", que permitan satisfacer las demandas de uso del recurso (Figueroa et al. 2003). De ahí, que es conveniente utilizar metodologías complementarias a las tradicionales, para determinar la calidad de las aguas y, en ese sentido, es indispensable poner en práctica los conceptos de bioindicación, evaluación biológica e índices de calidad, en cuanto a aguas se refiere.

La evaluación de la calidad del agua, se ha realizado, tradicionalmente, con base en los análisis fisicoquímicos y bacteriológicos (Madera et al. 2016; Roldán, 2016); sin embargo, en los últimos años, muchos países han aceptado la inclusión de las comunidades acuáticas, principalmente insectos, en la evaluación de la calidad de los ecosistemas acuáticos (Roldán, 2003; Prat et al. 2009; Roldán, 2016), debido a que los aspectos fisicoquímicos con los que por tradición se evalúan, solo dan una idea puntual sobre la calidad del ambiente y no ofrecen información sobre las variaciones en el tiempo (Alba-Tercedor, 1996; Springer, 2010).

Los insectos acuáticos son los organismos más ampliamente usados como bioindicadores, por diversas razones (Resh, 2008), entre las que se destacan, el tener una amplia distribución geográfica, una gran riqueza de especies con gran diversidad de respuestas a los gradientes ambientales, ser en su mayoría sedentarios, la posibilidad de utilizar su reacción de huida o deriva como indicador de contaminación y tener ciclos de vida largos, que permiten integrar los efectos de la contaminación en el tiempo (Prat et al. 2009; TrigalDomínguez et al. 2009). De otro lado, los integrantes de esta comunidad son sensibles a la contaminación orgánica y la degradación del hábitat, por tal razón, en la evaluación ambiental del recurso hídrico es valioso su potencial como bioindicadores de calidad de agua (Merritt \& Cummins, 1996).

La riqueza de insectos, la compleja red de interacciones que establecen con otros organismos y su característica de rápida respuesta ante las perturbaciones del medio, hacen de este grupo uno de los mejores bioindicadores del estado y funcionamiento del ecosistema, debido a que, muchos de ellos, tienen diferentes tolerancias a perturbaciones de su ecosistema (Sánchez et al. 2006).

La presencia y abundancia de los organismos bioindicadores es expresa mediante índices denominados "bióticos", los cuales, tienen la ventaja de tomar en cuenta la composición, mediante la ponderación, de acuerdo a la sensibilidad que presentan al factor de perturbación que se quiera valorar (Segnini, 2003).

En Colombia, se han realizado algunas investigaciones que determinan la diversidad de la entomofauna acuática, así como su uso en la determinación de la calidad del agua en diversos sistemas acuáticos, pudiendo mencionar, entre las más recientes, las de Meza et al. (2012), Vásquez \& Reinoso (2012), Ramírez et al. (2013), Roldán et al. (2014), Zúñiga et al. (2013; 2014), Zamora (2015), entre otras. En el caso del departamento del Chocó, el conocimiento sobre la diversidad de la entomofauna acuática es aun escaso, siendo los únicos trabajos publicados los de Torres et al. (2006), Mosquera et al. (2006), Córdoba et al. (2007), Salas et al. (2011) y Mosquera \& Córdoba (2015) y lo mismo sucede en cuanto a su uso en evaluación de calidad de agua, con solo dos trabajos publicados (Rivas et al. 2003; Asprilla et al. 2006).

Dada la escasez de información sobre calidad de agua, con base en la entomofauna acuática, así como la diversidad de esta comunidad en la región del Chocó, es prioritaria la realización de este tipo de investigaciones, teniendo en cuenta la alta diversidad que se ha reconocido y que posee la zona del Chocó, para diferentes grupos de insectos acuáticos (Mosquera \& Córdoba 2015; González et al. 2016; Rozo \& Salinas, 2016). Por esto, el objetivo de este estudio fue determinar la diversidad y la abundancia de los insectos acuáticos y la calidad del agua en diferentes quebradas, pertenecientes a la cuenca del río San Juan (Chocó), como una manera de proveer información específica sobre la diversidad y la riqueza de insectos acuáticos, que presentan estas quebradas, además de evaluar su calidad, con base en la diversidad y abundancia de esta comunidad biológica, contribuyendo a la generación de una línea de investigaciones de referencia para futuros programas de conservación, teniendo en cuenta los impactos sobre estas fuentes hídricas, como resultado de la actividad minera, al ser ésta, una de las principales vocaciones de la región.

\section{MATERIALES Y MÉTODOS}

Área de estudio. La zona de estudio, se encuentra localizada en los municipios de Tadó y Unión Panamericana, ubicados en la cuenca media del río San Juan, en el departamento del Chocó, Colombia (Figura 1 y 2). Esta cuenca, se ubica entre los $5^{\circ} 16^{\prime} \mathrm{N}$ y $76^{\circ} 73^{\circ} \mathrm{O}$, presenta una temperatura de entre los 
27 y $29^{\circ} \mathrm{C}$ y una precipitación anual promedio, que oscila entre los 6.800 y $7.600 \mathrm{~mm}$, a una altura de $75 \mathrm{msnm}$ y pertenece a la zona de vida de Bosque Pluvial Tropical (bp-T) (Holdridge, 1996). En la tabla 1, se describen las características de las cinco quebradas seleccionadas en la zona de estudio.
Comunidad de insectos acuáticos: Se realizaron cuatro muestreos entre abril y agosto de 2015. Para la colecta de los organismos, se seleccionó un tramo representativo de $20 \mathrm{~m}^{2}$ en cada una de las quebradas objeto de estudio (Roldán, 2003), empleando diferentes métodos, de acuerdo al sustrato presente: una red D-net, para la vegetación margi-

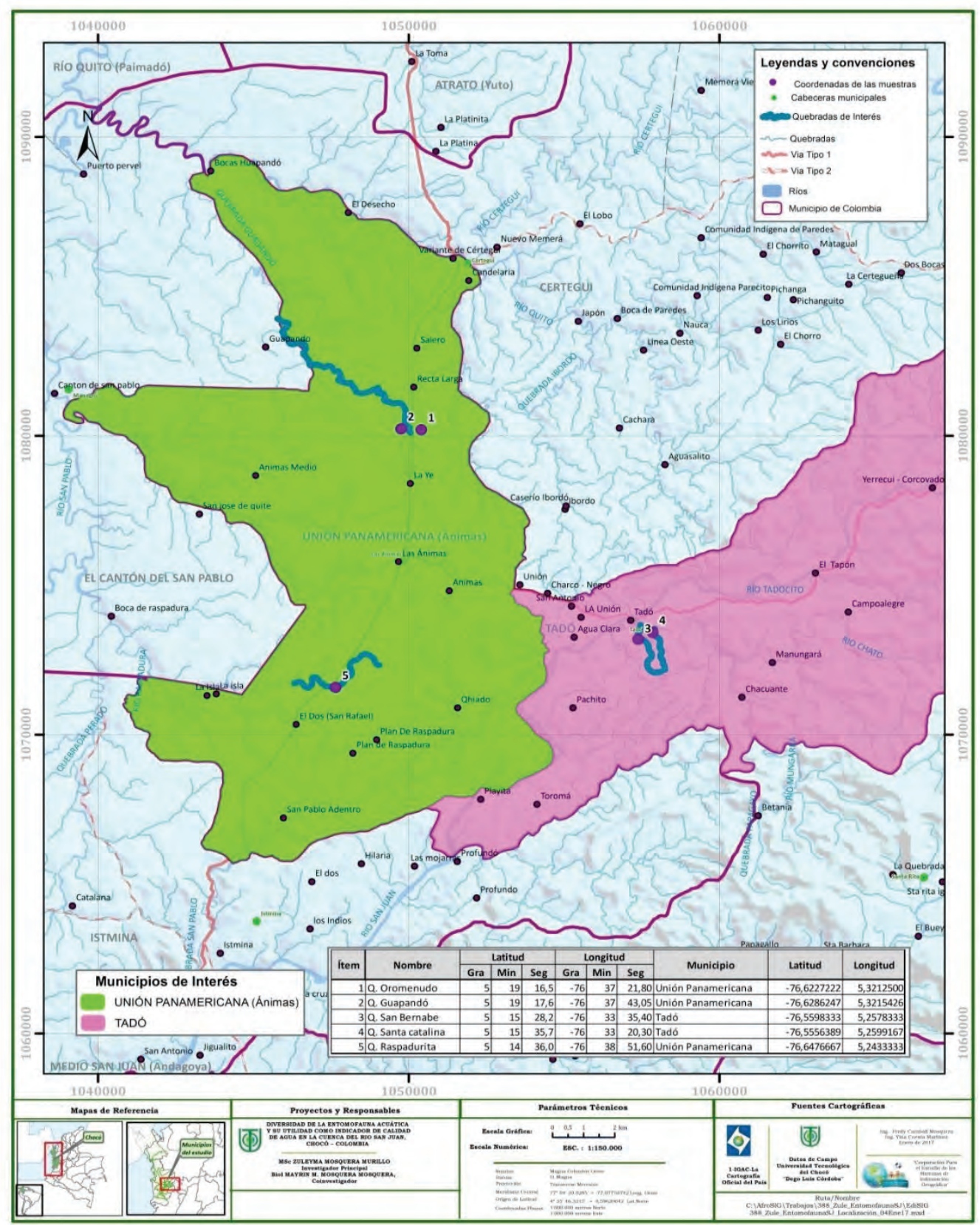

Figura 1. Localización de las quebradas estudiadas en la zona del San Juan, Chocó. 


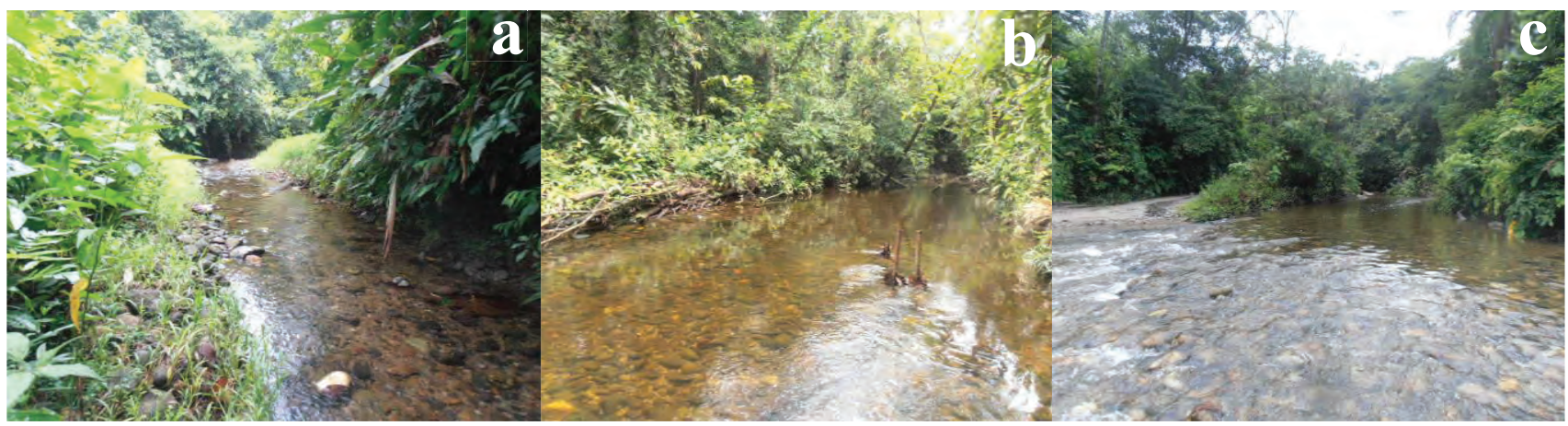

Figura 2. Quebradas de la cuenca del río San Juan: a) Quebrada Santa Catalina; b) Quebrada Guapandó; c) Quebrada Raspadurita. Fotos: Zuleyma Mosquera-Murillo.

Tabla 1. Descripción de las quebradas estudiadas en la cueca media del río San Juan, Chocó - Colombia.

\begin{tabular}{|c|c|c|}
\hline Quebrada & $\begin{array}{c}\text { Coordenadas y } \\
\text { Altitud }\end{array}$ & Descripción \\
\hline $\begin{array}{l}\text { Quebrada } \\
\text { Oromenudo }\end{array}$ & $\begin{array}{l}\text { 5०19' LN } \\
76^{\circ} 37^{\prime} \text { LO } \\
130 \text { msnm }\end{array}$ & $\begin{array}{l}\text { Ancho promedio de } 1,44 \mathrm{~m} \text { y profundidad de } 0,28 \mathrm{~m} \text {. Temperatura del agua prome- } \\
\text { dio de } 26,35^{\circ} \mathrm{C} \text {, velocidad de } 0,23 \mathrm{~m} / \mathrm{s} \text { y caudal de } 0,080 \mathrm{~m}^{3} / \mathrm{s} \text {. Sustrato cubierto por } \\
\text { arena, piedras y hojarasca. La vegetación es la asociada a ambientes de terrazas de } \\
\text { material rocoso, cubierta por epifitos (musgos y hepáticas), junto a especies de hel- } \\
\text { echos, Cyclanthaceas e individuos herbáceos de Rubiaceae. }\end{array}$ \\
\hline $\begin{array}{l}\text { Quebrada } \\
\text { Guapando }\end{array}$ & $\begin{array}{l}\text { 5¹9' LN } \\
76^{\circ} 34^{\prime} \text { LO } \\
\text { 103msnm }\end{array}$ & $\begin{array}{l}\text { Ancho promedio de } 6,6 \mathrm{~m} \text { y profundidad de } 0,31 \mathrm{~m} \text {. Temperatura del agua promedio } \\
\text { de } 26,80^{\circ} \mathrm{C} \text {, velocidad de } 0,44 \mathrm{~m} / \mathrm{s} \text { y caudal de } 0,85 \mathrm{~m}^{3} / \mathrm{s} \text {. Sustrato cubierto por pie- } \\
\text { dras y guijarros. Vegetación de llanura aluvial con bosque medianamente intervenido, } \\
\text { representado a nivel de ribera por las familias Moraceae, Rubiaceae, Acanthaceae, } \\
\text { Myristicaceae, Arecaceae y a nivel del suelo por Cyclanthaceae y Pteridofitos (hel- } \\
\text { echos) (Figura 2). }\end{array}$ \\
\hline $\begin{array}{c}\text { Quebrada } \\
\text { Santa Catalina }\end{array}$ & $\begin{array}{l}\text { 5०15' LN } \\
76^{\circ} 33^{\prime} \mathrm{LO} \\
95 \mathrm{msnm}\end{array}$ & $\begin{array}{l}\text { Ancho promedio de } 3,03 \mathrm{~m} \text { y profundidad de } 0,32 \mathrm{~m} \text {. Temperatura del agua de } \\
27,57^{\circ} \mathrm{C} \text {, velocidad de } 0,31 \mathrm{~m} / \mathrm{s} \text { y caudal de } 0,27 \mathrm{~m}^{3} / \mathrm{s} \text {. Sustrato cubierto por arena y } \\
\text { piedras. Vegetación de llanura aluvial, con bosque medianamente intervenido, repre- } \\
\text { sentado por las familias Rubiaceae, Melastomataceae (Miconia sp.), Heliconiaceae, } \\
\text { Araceae, Dioscoreaceae, Acanthaceae y gramíneas (Paspalum sp.) (Figura 2). }\end{array}$ \\
\hline $\begin{array}{l}\text { Quebrada } \\
\text { Raspadurita }\end{array}$ & $\begin{array}{l}, 5^{\circ} 14^{\prime} \text { LN } \\
76^{\circ} 38^{\prime} \text { LO } \\
133 \mathrm{msnm}\end{array}$ & $\begin{array}{l}\text { Ancho promedio de } 11,64 \mathrm{~m} \text { y profundidad de } 0,46 \mathrm{~m} \text {. Temperatura del agua de } \\
26,30^{\circ} \mathrm{C} \text {, velocidad de } 0,49 \mathrm{~m} / \mathrm{s} \text { y caudal de } 2,52 \mathrm{~m}^{3} / \mathrm{s} \text {. Sustrato cubierto por piedras } \\
\text { de gran tamaño. Vegetación de llanura aluvial, bosque intervenido, con individuos } \\
\text { de porte arbóreo con algunas familias como Mimosaceae (Pichinde), Myrtaceae, Ar- } \\
\text { aceae, Helechos (Sticherus sp. y Gleichenia sp.) y algunas gramíneas (Figura 2). }\end{array}$ \\
\hline $\begin{array}{l}\text { Quebrada } \\
\text { San Bernabé }\end{array}$ & $\begin{array}{l}5^{\circ} 15^{\prime} \mathrm{LN} \\
76^{\circ} 33^{\prime} \text { LO } \\
94 \mathrm{msnm}\end{array}$ & $\begin{array}{l}\text { Ancho promedio de } 4,97 \mathrm{~m} \text { y profundidad de } 0,35 \mathrm{~m} \text {. Temperatura del agua de } \\
26,37^{\circ} \mathrm{C} \text {, velocidad de } 0,39 \mathrm{~m} / \mathrm{s} \text { y caudal de } 0,63 \mathrm{~m}^{3} / \mathrm{s} \text {. Sustrato cubierto por piedras y } \\
\text { arena. Vegetación de llanura aluvial con bosque altamente intervenido. Se distinguen } \\
\text { en sus riberas las familias Melastomataceae (Miconia sp.), Helechos (Sticherus sp. } \\
\text { y Gleichenia sp.), Araceae, Gramineas (Paspalum sp.), Myristicaceae, Arecaceae y } \\
\text { Rubiaceae. }\end{array}$ \\
\hline
\end{tabular}


nal y pinzas entomológicas, para los organismos presentes en piedras, en troncos y en hojarasca. Se utilizó, igual esfuerzo de muestreo, en cada uno de los sustratos presentes: piedra, hojarasca, vegetación ribereña y troncos (30minutos), con dos réplicas para cada uno. Aunque cada muestra se procesó por separado, para los análisis los registros de abundancia fueron promediados y considerados como un solo dato, para cada fecha y quebrada. Los organismos colectados fueron fijados en alcohol al $70 \%$ y transportados al Laboratorio de Limnología de la Universidad Tecnológica del Chocó, donde se realizó la determinación taxonómica de los especímenes hasta género, utilizando las claves de Domínguez et al. (2006), Merritt et al. (2008), Domínguez \& Fernández (2009), Springer et al. (2010). Los ejemplares, se encuentran depositados en la Colección Limnológica del Chocó "CLCH".

Calidad de agua de las quebradas: Para la evaluación de calidad del agua en cada una de las quebradas estudiadas, se utilizó el índice BMWP (Biological Monitoring Working Party), adaptado para Colombia (BMWP/Col.), que se basa en algunas familias de macroinvertebrados acuáticos, cuyos puntajes individuales reflejan su tolerancia a la contaminación. El índice, se obtiene por la suma total de la puntuación de cada familia (1-10) y define clases, calidad, puntaje y categorías (Roldán, 2003). Igualmente, se calculó el índice ASPT (Average Score Per Taxon= Puntaje promedio por Taxon=1-10), que se obtiene al dividir el BMWP, por el número de taxa incluidos en el cálculo (Armitage et al. 1983).

En cada quebrada estudiada, se midieron in situ, con un equipo digital multiparámetro (HACH HQ30d), el oxígeno disuelto, el $\mathrm{pH}$, la temperatura del agua, la conductividad eléctrica y los sólidos totales disueltos; la alcalinidad total, se midió siguiendo las recomendaciones de Standard Methods (APHA, 2012), en el Laboratorio de Limnología de la Universidad Tecnológica del Chocó. Además, se tomaron muestras de agua para ser analizadas en el laboratorio y determinar los nitritos, los nitratos, el amonio y los fosfatos.

Análisis de datos: La diversidad de la comunidad de insectos acuáticos, se estimó mediante el índice de diversidad de Shannon - Weaver, el índice de dominancia de Simpson y la riqueza específica (programa Past versión 1.57, Hammer et al. 2001). Para comparar la composición, a nivel de géneros, de la comunidad de insectos acuáticos entre quebradas, se realizó un análisis Cluster, utilizando el índice de similitud de Bray-Curtis. Las diferencias en diversidad, en dominancia y en riqueza específica entre quebradas fueron evaluadas mediante un análisis de varianza (ANOVA una vía). La calidad de agua de las quebradas, se evaluó con los índices BMWP/Col (Roldán, 2003) y ASPT (Average Score Per Taxon, Armitage \& Petts, 1992). Adicionalmente, se eje- cutó una prueba de correlación múltiple entre la estructura de la comunidad de insectos acuáticos, los valores de los índices BMWP/Col, ASPT y las variables fisicoquímicas. Todos los análisis estadísticos mencionados fueron realizados mediante los programas StatGraphics versión 5.1.

\section{RESULTADOS Y DISCUSIÓN}

Diversidad de insectos acuáticos: Se colectaron 4.100 individuos, distribuidos en nueve órdenes, 37 familias y 59 géneros (Tabla 2). En la quebrada Guapandó, se colectaron 1.678 individuos, en 30 familias y 43 géneros, de los cuales, 3 fueron exclusivos de este ecosistema: Marilia, Laccophilus, Maruina y Georissus. En la quebrada Raspadurita, 1.405 individuos, en 26 familias y 46 géneros, donde Tricorythopsis es exclusivo de esta quebrada. En la quebrada Santa Catalina, se colectaron 513 individuos, en 23 familias y 38 géneros, de los cuales, 3 fueron exclusivos de esta quebrada: Macrostenum y Helichus. En la quebrada San Bernabé, 376 individuos, en 23 familias y 35 géneros, siendo Simulium exclusivo de esta quebrada. En la quebrada Oromenudo, se colectaron 128 individuos en 17 familias y 29 géneros, donde Ranatra fue exclusivo de esta quebrada. En cuanto a la composición, se encontró que el 37,11\% de los géneros registrados (16) son compartidos por las cinco quebradas estudiadas, entre ellos Cylloepus, Macrelmis, Smicridea, Argia, Psephenus, Corydalus, Anacroneuria, Farrodes, Thraulodes, Americabaetis y Tikuna; este último ha sido reportado, recientemente, por segunda vez para el país, por Mosquera \& Córdoba (en prensa) (Tabla 2) (Figura 3).

A nivel de órdenes, Ephemeroptera fue el más representativo, tanto en términos de abundancia, con 1.407 organismos (34,32\%), como de riqueza específica (13 géneros), seguido de Trichoptera, con 1.026 organismos $(25,02 \%)$ y Odonata, con $745(18,17 \%)$ y, en menor proporción, se registra Lepidoptera, con 2 organismos (0,05\%) (Tabla 2).

La composición de la comunidad de insectos acuáticos en esta investigación es similar a la reportada por Mosquera \& Córdoba (2015) en otras quebradas, ubicadas en la misma zona de estudio, con prevalencia de los órdenes Ephemeroptera y Trichoptera. Particularmente, el grupo de los efemerópteros, sobresale en todas las quebradas, lo cual, se relaciona con los múltiples ciclos de reproducción en el año (polivoltinos) y su capacidad de colonizar diferentes sustratos, presentando mayor diversidad en ríos de aguas bien oxigenadas, con fondo rocoso, al ser capaces de vivir en casi todo tipo de cuerpos dulceacuícolas (Prat et al. 2009).

Las diferencias en abundancia y en composición de la comunidad de insectos acuáticos observadas entre las quebradas, se puede atribuir a las diferencias en morfología que genera cambios en el tipo y disponibilidad de los sustratos, vegeta- 
Tabla 2. Insectos acuáticos colectados en las quebradas estudiadas.

\begin{tabular}{|c|c|c|c|c|c|c|c|c|c|}
\hline ORDEN & FAMILIA & GÉNERO & 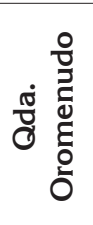 & 它 & 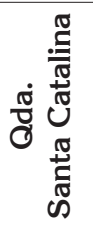 & 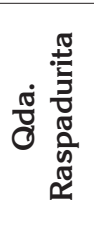 & 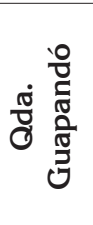 & 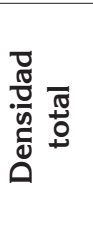 & 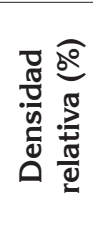 \\
\hline \multirow{13}{*}{ Ephemeroptera } & \multirow{4}{*}{ Baetidae } & Americabaetis & 7 & 19 & 13 & 10 & 41 & 90 & 2,20 \\
\hline & & Cloeodes & 10 & 42 & 9 & 14 & 55 & 130 & 3,17 \\
\hline & & Baetodes & 4 & 25 & 52 & 33 & 34 & 148 & 3,61 \\
\hline & & Camelobaetidius & 2 & 0 & 30 & 31 & 23 & 86 & 2,10 \\
\hline & \multirow{6}{*}{ Leptophlebiidae } & Miroculis & 6 & 2 & 0 & 2 & 7 & 17 & 0,41 \\
\hline & & Farrodes & 8 & 18 & 13 & 31 & 1 & 71 & 1,73 \\
\hline & & Thraulodes & 15 & 8 & 56 & 46 & 70 & 195 & 4,76 \\
\hline & & Terpides & 10 & 5 & 11 & 33 & 9 & 68 & 1,66 \\
\hline & & Tikuna & 4 & 28 & 7 & 134 & 60 & 233 & 5,68 \\
\hline & & Hydrosmilodon & 2 & 0 & 80 & 90 & 0 & 172 & 4,20 \\
\hline & \multirow{2}{*}{ Leptohyphidae } & Tricorythopsis & 0 & 0 & 0 & 16 & 0 & 16 & 0,39 \\
\hline & & Leptohyphes & 0 & 1 & 0 & 178 & 0 & 179 & 4,37 \\
\hline & Euthyplociidae & Campylocia & 1 & 0 & 0 & 1 & 0 & 2 & 0,05 \\
\hline \multirow{11}{*}{ Trichoptera } & Odontoceridae & Marilia & 0 & 0 & 0 & 0 & 1 & 1 & 0,02 \\
\hline & Leptoceridae & Nectopsyche & 4 & 0 & 2 & 19 & 16 & 41 & 1,00 \\
\hline & \multirow{4}{*}{ Hydropsychidae } & Smicridea & 1 & 4 & 16 & 117 & 694 & 832 & 20,29 \\
\hline & & Macrostemum & 0 & 0 & 1 & 0 & 0 & 1 & 0,02 \\
\hline & & Macronema & 4 & 0 & 0 & 1 & 0 & 5 & 0,12 \\
\hline & & Leptonema & 1 & 0 & 1 & 3 & 0 & 5 & 0,12 \\
\hline & Glossosomatidae & Culoptila & 0 & 0 & 0 & 3 & 69 & 72 & 1,76 \\
\hline & Calamoceratidae & Phylloicus & 0 & 0 & 0 & 2 & 1 & 3 & 0,07 \\
\hline & \multirow{2}{*}{ Polycetropodidae } & Poliplectropus & 0 & 3 & 3 & 3 & 3 & 12 & 0,29 \\
\hline & & Cyrnellus & 2 & 3 & 0 & 1 & 0 & 6 & 0,15 \\
\hline & Phylopotamidae & Chimarra & 2 & 10 & 29 & 4 & 3 & 48 & 1,17 \\
\hline Plecoptera & Perlidae & Anacroneuria & 3 & 9 & 3 & 162 & 2 & 179 & 4,37 \\
\hline \multirow{12}{*}{ Odonata } & Calopterygidae & Hetaerina & 0 & 24 & 32 & 25 & 8 & 89 & 2,17 \\
\hline & Platystictidae & Palaemnema & 0 & 0 & 6 & 10 & 29 & 45 & 1,10 \\
\hline & \multirow{3}{*}{ Libellulidae } & Perithemis & 8 & 25 & 33 & 27 & 19 & 112 & 2,73 \\
\hline & & Dythemis & 2 & 7 & 7 & 12 & 33 & 61 & 1,49 \\
\hline & & Elasmothemis & 4 & 0 & 1 & 4 & 15 & 24 & 0,59 \\
\hline & Politoridae & Polythore & 4 & 2 & 1 & 8 & 20 & 35 & 0,85 \\
\hline & Cordulidae & Neocordulia & 0 & 4 & 0 & 0 & 10 & 14 & 0,34 \\
\hline & Coenagrionidae & Argia & 9 & 16 & 17 & 35 & 67 & 144 & 3,51 \\
\hline & Perilestidae & Perissolestes & 4 & 1 & 0 & 0 & 0 & 5 & 0,12 \\
\hline & \multirow{2}{*}{ Gomphidae } & Desmoghomphus & 0 & 18 & 24 & 33 & 127 & 202 & 4,93 \\
\hline & & Styloghomphus & 0 & 0 & 0 & 1 & 1 & 2 & 0,05 \\
\hline & Megapodagrionidae & Heteragrion & 0 & 0 & 6 & 2 & 4 & 12 & 0,29 \\
\hline \multirow{6}{*}{ Hemiptera } & \multirow{4}{*}{ Naucoridae } & Cryphocricos & 0 & 3 & 2 & 7 & 4 & 16 & 0,39 \\
\hline & & Limnocoris & 0 & 2 & 3 & 0 & 0 & 5 & 0,12 \\
\hline & & Ambrysus & 0 & 1 & 4 & 9 & 7 & 21 & 0,51 \\
\hline & & Pelocoris & 0 & 9 & 5 & 8 & 3 & 25 & 0,61 \\
\hline & Nepidae & Ranatra & 2 & 0 & 0 & 0 & 0 & 2 & 0,05 \\
\hline & Veliidae & Rhagovelia & 1 & 14 & 6 & 3 & 2 & 26 & 0,63 \\
\hline
\end{tabular}


Continuación Tabla 2.

\begin{tabular}{|c|c|c|c|c|c|c|c|c|c|}
\hline \multirow{9}{*}{ Coleoptera } & Gyrinidae & Gyretes & 1 & 11 & 0 & 0 & 0 & 12 & 0,29 \\
\hline & Ptilodactilidae & Anchytarsus & 0 & 16 & 6 & 2 & 2 & 26 & 0,63 \\
\hline & \multirow{3}{*}{ Elmidae } & Cylloepus & 0 & 8 & 17 & 28 & 37 & 90 & 2,20 \\
\hline & & Microcylloepus & 0 & 0 & 1 & 1 & 0 & 2 & 0,05 \\
\hline & & Macrelmis & 2 & 3 & 0 & 68 & 10 & 83 & 2,02 \\
\hline & Dryopidae & Helichus & 0 & 0 & 2 & 0 & 0 & 2 & 0,05 \\
\hline & Psephenidae & Psephenus & 5 & 3 & 4 & 88 & 40 & 140 & 3,41 \\
\hline & Dytiscidae & Laccophilus & 0 & 0 & 0 & 0 & 2 & 2 & 0,05 \\
\hline & Georissidae & Georisuss & 0 & 0 & 0 & 0 & 6 & 6 & 0,15 \\
\hline \multirow{5}{*}{ Diptera } & \multirow{2}{*}{ Chironomidae } & Pentaneura & 0 & 0 & 2 & 1 & 0 & 3 & 0,07 \\
\hline & & Ablabesmyia & 0 & 0 & 0 & 12 & 48 & 60 & 1,46 \\
\hline & Tipulidae & Hexatoma & 0 & 1 & 1 & 11 & 35 & 48 & 1,17 \\
\hline & Psychodidae & Maruina & 0 & 0 & 0 & 0 & 1 & 1 & 0,02 \\
\hline & Simulidae & Simulium & 0 & 2 & 0 & 0 & 0 & 2 & 0,05 \\
\hline Lepidoptera & Crambridae & Petrophila & 0 & 0 & 0 & 0 & 2 & 2 & 0,05 \\
\hline Megaloptera & Corydalidae & Corydalus & 0 & 29 & 7 & 76 & 57 & 169 & 4,12 \\
\hline \multicolumn{3}{|c|}{ Abundancia Total } & 128 & 376 & 513 & 1405 & 1678 & 4100 & \\
\hline \multicolumn{3}{|c|}{ Puntaje BMWP/Col } & 129 & 165 & 174 & 190 & 198 & & \\
\hline \multicolumn{3}{|l|}{ ASPT } & 8,06 & 7,85 & 7,56 & 7,6 & 7,61 & & \\
\hline
\end{tabular}
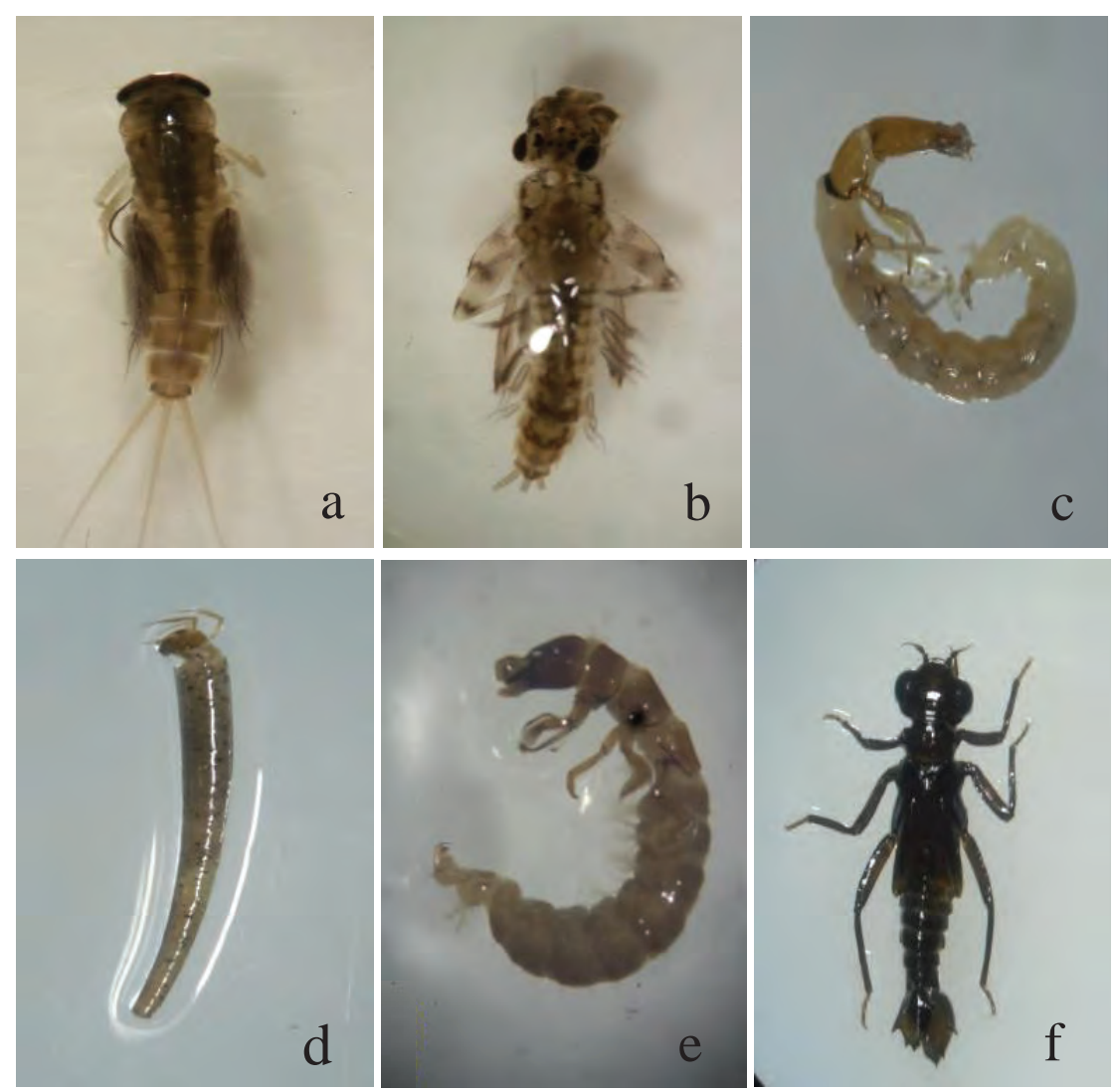

Figura 3. Detalle de algunos géneros registrados: a) Tikuna (Ephemeroptera); b) Miroculis (Ephemeroptera); c) Chimarra (Trichoptera); d) Nectopsiche (Trichoptera); e) Macrostemum (Trichoptera); f) Polythore (Odonata). Fuente: Z. Mosquera. 
ción ribereña, cauce, velocidad de la corriente, así como en las condiciones fisicoquímicas de las mismas, ya que, en general, los macroinvertebrados que viven en ecosistemas lóticos (ríos, arroyos, quebradas), se acomodan en un gradiente longitudinal y transversal en relación con la velocidad de la corriente y de la colonización de los diferentes microhábitats, por lo tanto, existe un mosaico faunístico correspondiente con el mosaico de tipos de sedimentos depositados sobre el lecho del río y, en consecuencia, se puede esperar una mayor abundancia de organismos en aquellos tramos con mayor heterogeneidad del sustrato y, por el contrario, allí donde el sustrato sea uniforme o exista una mayor homogeneidad del lecho del río, la abundancia esperada será menor (Segnini, 2003; Arango et al. 2008), situación que se presenta en el presente estudio.

Los resultados de los índices ecológicos fueron homogéneos entre quebradas, como lo demuestra el Análisis de Varianza, con p>0,05, en todos los casos (Figura 4). El índice de diversidad Shannon-Weaver ( $\left.\mathrm{H}^{\prime}\right)$ presentó su máximo valor en la quebrada San Bernabé, con rangos entre 3,14 y 2,52bits/ind. La dominancia de Simpson presentó rangos entre 0,94 y 0,80, entre tanto, la riqueza específica fue mayor en Raspadurita, con un rango de entre 29 y 46 géneros.
Considerando que los valores $\mathrm{H}^{\prime} \geq 2,70$ comprenden alta diversidad y H' entre 1,50-2,70, corresponden a diversidad media (Margalef, 1983), se puede clasificar a las quebradas Santa Catalina, Oromenudo, San Bernabé y Raspadurita, como correspondientes a diversidad alta, mientras que la diversidad es media para la quebrada Guapandó. Estos resultados son explicados por la variedad de sustratos, que se encontraron presentes en las mismas, los que estuvieron constituidos, principalmente, por piedras, por hojarasca, por sedimento y por vegetación ribereña (Figura 2), lo que sugiere, una mayor variedad de fuentes de alimento y de hábitos alimenticios, que brindan estabilidad para la colonización, la alimentación, el refugio y la reproducción de un gran número de invertebrados acuáticos (Chará, 2003). Asimismo, en estos lugares existió una buena cobertura vegetal en la zona de ribera que, en cierta forma, mejora el hábitat (Cárdenas et al. 2007), puesto que contribuye a tener mejor calidad de agua, mayor integridad del cauce, mejor hábitat físico y, consecuentemente, una biota más diversa dentro de las quebradas (Chará et al. 2007). Los valores altos de dominancia, asociados a la alta abundancia que presentaron (Holzenthal et al. 2007; Zúñiga et al. 2014) han sido reportados como abundantes en otras investigaciones realizadas en la zona, como las de Asprilla et al. (2006), Córdoba et al. (2007) y Mosquera \& Córdoba (2015).
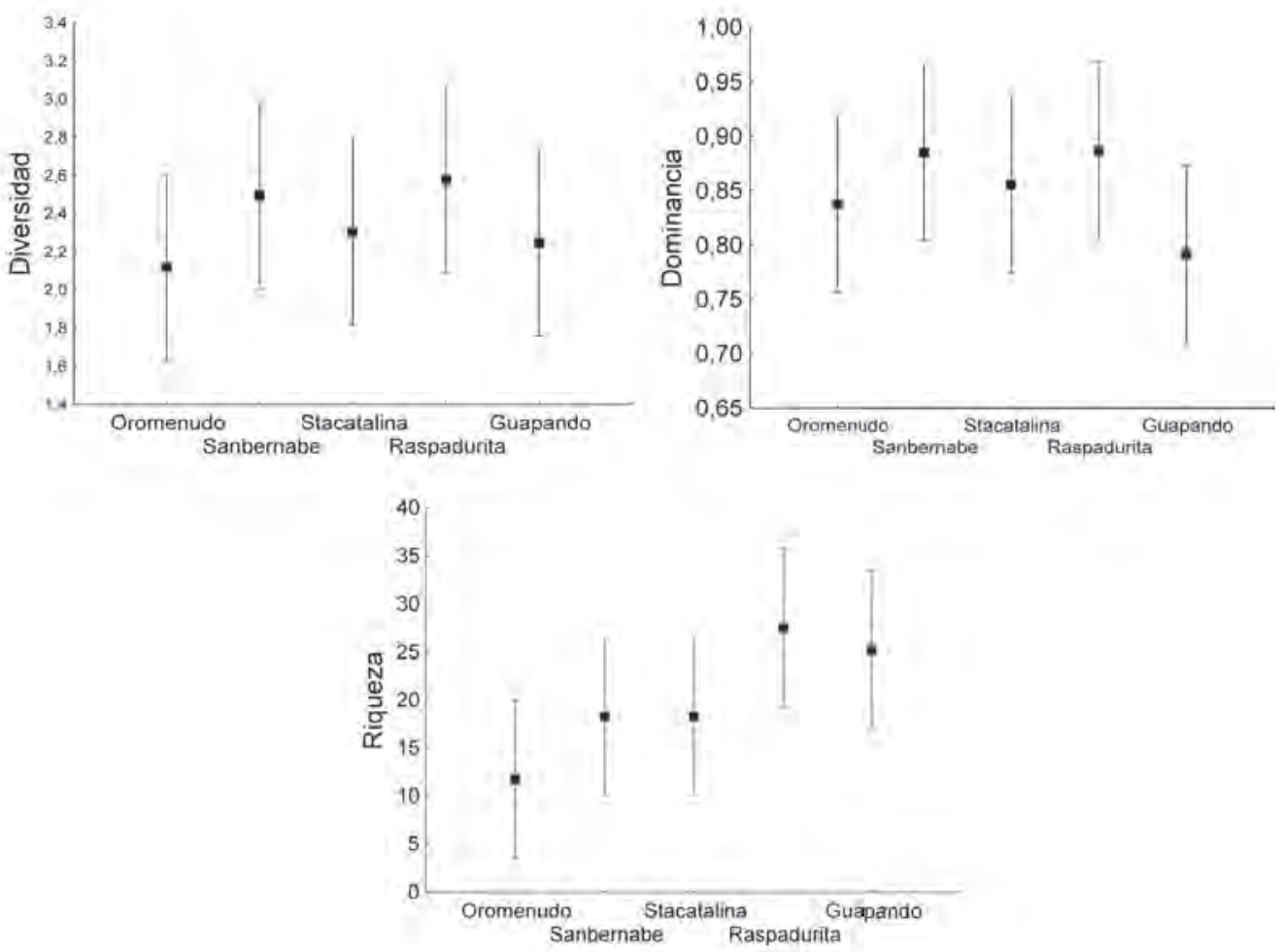

Figura 4. Gráfico de medias para la diversidad, dominancia y riqueza específica de macroinvertebrados acuáticos en las quebradas estudiadas. 
Al analizar la similaridad entre quebradas, se observa la formación de dos grupos bien definidos (Figura 5), un primer grupo formado por las quebradas Guapandó y Raspadurita y otro por Santa Catalina, San Bernabé y Oromenudo, con la más alta similaridad, observada entre las quebradas San Bernabé y Santa Catalina, con un 51,84\%, mientras que las más disimiles son Oromenudo y Raspadurita, con un 21,92\% (Figura 5).

Los valores de similitud observados, con la mayor similitud entre las quebradas Santa Catalina y San Bernabé, son atribuibles a su cercanía, al encontrarse ambas en el casco urbano del municipio de Tadó (Chocó), lo que generó condiciones similares de hábitat y de cobertura boscosa, entre estos dos ecosistemas, los cuales, son factores importantes para los macroinvertebrados acuáticos, como ha sido señalado por diversos autores (Alonso, 2006; Arango et al. 2008), quienes resaltan la importancia de la vegetación ribereña en la composición, la riqueza y la abundancia de macroinvertebrados acuáticos, al proveer heterogeneidad de hábitat para el establecimiento de la mayoría de grupos de macroinvertebrados (Richardson \& Moore, 2010); igualmente, estas quebradas comparten un importante número de familias y de géneros, aunque no se pueden descartar otros factores, como la temperatura del agua, las adaptaciones fisiológicas de los organismos, la amplitud de los cauces, los niveles de oxígeno y la corriente de las quebradas, factores que influyen sobre la comunidad de macroinvertebrados acuáticos y, entre ellos, los insectos (Roldán, 2003).

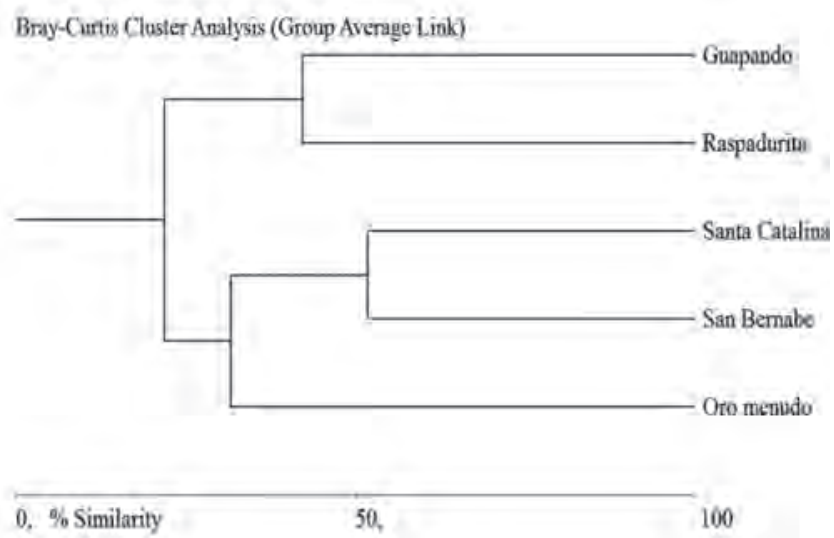

Figura 5. Similitud de la composición de géneros en las quebradas estudiadas.

Calidad de agua de las quebradas: La calidad del agua de las cinco quebradas, se evaluó con el índice BMWP'/Col (Roldán, 2003), encontrándose que los valores oscilaron entre 129, para la quebrada Oromenudo y 198, para Guapandó (Tabla 2), indicando que en los ecosistemas estudiados las aguas son muy limpias, con calidad buena o no alteradas, de manera sensible, a aguas de calidad excelente (valores entre 101-150 a >150), de acuerdo con la escala publicada por Roldán (2003). Por otro lado, de acuerdo con el ASPT, casi todas las quebradas tienen agua Clase II, aceptable (ligeramente contaminada); sin embargo, la quebrada Oromenudo presentó aguas Clase I, buena (aguas no contaminadas) (Tabla 2).

Estos resultados son similares a los reportados por García et al. (2012), quienes encontraron ligeras diferencias en los resultados arrojados por los índices BMWP/Col y ASPT, atribuibles a que este último es más sensible a la intervención antrópica, al incluir en su cálculo la riqueza de familias; sin embargo, es importante resaltar que estos dos índices están estrechamente relacionados, lo que es corroborado por los trabajos de Arango et al. (2008), Bustamante et al.
(2011) y Ramírez et al. (2013), quienes reportan cómo la utilización conjunta de estos dos índices, brinda una información sólida para definir la calidad del agua en ecosistemas sujetos a variados tipos de perturbación antrópica.

En cuanto a las variables fisicoquímicas, los promedios de cada quebrada se muestran en la tabla 3. Se encontró que en promedio la concentración de oxígeno disuelto, el $\mathrm{pH}$, la temperatura, la alcalinidad y la conductividad eléctrica registraron sus mayores valores en la quebrada Santa Catalina. El menor promedio de oxígeno y de conductividad, se registra en Oromenudo, mientras que en Raspadurita, se consignan los valores más bajos de $\mathrm{pH}$, de temperatura y de alcalinidad. En el caso de los nutrientes, tienden a ser mayores en Raspadurita (Nitritos y Nitratos), Guapando (Amonio) y Santa Catalina (Fosfatos). De acuerdo al análisis de correlación múltiple, se observó que el índice BMWP/Col, se encuentra correlacionado, positivamente, con la diversidad y la riqueza de insectos acuáticos; en tanto que, en cuanto a la estructura, los índices de diversidad, de riqueza y de dominancia mostraron correlaciones positivase entre ellos (Tabla 4). 
Los factores fisicoquímicos son considerados, por algunos autores, como los aspectos que más influencia ejercen sobre la distribución, la abundancia y la riqueza de insectos acuáticos (Shieh \& Yang, 2000; Ocon et al. 2004). En el caso de las quebradas estudiadas, los parámetros medidos se hallan dentro de los rangos normales, para este tipo de ambientes, coincidiendo con otras investigaciones realizadas en el Chocó, como las de Salas et al. (2011), Contreras-Martínez (2013), Mosquera \& Córdoba (2015), a excepción de los fosfatos, que se encuentran por encima del rango requerido para la vida acuática en aguas naturales, que es de $0,05 \mathrm{mg} / \mathrm{L}$, lo que es resultado de la cercanía de algunas quebradas a algunos asentamientos urbanos del área; no obstante, son similares a los reportados por otras investigaciones adelantadas en la zona, como las de Mosquera \& Córdoba (2015). Valores normales de parámetros, como el oxígeno, el $\mathrm{pH}$, la temperatura y la conductividad son importantes en los ecosistemas acuáticos, ya que están relacionados con los procesos metabólicos de los organismos aeróbicos y la productividad biológica, respectivamente (Nieves et al. 2010).

Tabla 3. Variables fisicoquímicas para cada quebrada estudiada.

\begin{tabular}{|l|c|c|c|c|c|c|}
\hline \multirow{2}{*}{\multicolumn{1}{c|}{ Fisicoquímicos }} & \multicolumn{5}{c|}{ Quebradas } \\
\cline { 2 - 7 } & Oro menudo & San Bernabé & Santa catalina & Guapandó & Raspadurita & CV \\
\hline Oxígeno disuelto (mg/L) & 5,69 & 7,33 & 7,41 & 5,96 & 6,85 & 11,84 \\
\hline $\mathrm{pH}$ & 5,84 & 6,4 & 6,56 & 6,55 & 5,64 & 6,92 \\
\hline Temperatura del agua $\left({ }^{\circ} \mathrm{C}\right)$ & 26,35 & 26,37 & 27,57 & 26,8 & 26,2 & 2,01 \\
\hline Alcalinidad $(\mathrm{mg} / \mathrm{L} \mathrm{CaCO} 3)$ & 8,67 & 11,17 & 14,75 & 12,57 & 6,83 & 28,98 \\
\hline Conductividad $(\mu \mathrm{S} / \mathrm{cm})$ & 7,14 & 21,25 & 29,77 & 22,44 & 11,23 & 49,60 \\
\hline Nitritos $(\mathrm{mg} / \mathrm{L})$ & 0,13 & 0,02 & 0,01 & 0,19 & 0,27 & 89,73 \\
\hline Nitratos $(\mathrm{mg} / \mathrm{L})$ & 0,64 & 0,72 & 0,11 & 0,88 & 1,15 & 54,73 \\
\hline Amonio $(\mathrm{mg} / \mathrm{L})$ & 0,09 & 0,26 & 0,08 & 0,38 & 0,05 & 82,77 \\
\hline Fosfato $(\mathrm{mg} / \mathrm{L})$ & 0,05 & 0,05 & 0,22 & 0,07 & 0,09 & 74,24 \\
\hline
\end{tabular}

Tabla 4. Resultados significativos de la prueba de correlación múltiple entre la estructura de la comunidad de insectos acuáticos, los valores del índice BMWP/Col y las variables fisicoquímicas.

\begin{tabular}{|l|c|c|c|c|}
\hline \multirow{2}{*}{ Variable } & \multicolumn{2}{|c|}{ Diversidad } & \multicolumn{2}{c|}{ Riqueza } \\
\cline { 2 - 5 } & $\mathbf{r}$ & $\mathbf{p}$ & $\mathbf{r}$ & $\mathbf{p}$ \\
\hline BMWP/Col & 0,67 & 0,0033 & 0,93 & 0,0020 \\
\hline Diversidad & - & & 0,70 & 0,0023 \\
\hline Dominancia & 0,90 & 0,0010 & - & \\
\hline
\end{tabular}

Según este estudio, las quebradas evaluadas en la cuenca del río San Juan presentan una comunidad de insectos acuáticos muy diversa y compleja, lo que se asocia con sistemas que no han sido alterados o con poca intervención antropogénica, lo cual, se refleja en los resultados arrojados por los índices bióticos, basados en esta comunidad biológica; sin embargo, es importante seguir adelantando estudios de monitoreo en la zona, teniendo en cuenta las amenazas a las que se enfrentan estos ecosistemas en la actualidad, como resultado del avance en la actividad minera y de explotación maderera en el área.

Agradecimientos: Las autoras agradecen a la Fundación para la Promoción de la Investigación y la Tecnología del Banco de la República, por la financiación de esta investigación y a la Universidad Tecnológica del Chocó, por faci- litar la infraestructura de campo y laboratorios. A las biólogas Yasiris Salas Tovar (Q.E.P.D.), Sandra Patricia Sánchez y Karen Y. Palomeque, por su apoyo en la fase de campo de esta investigación. Agradecemos igualmente, a dos revisores anónimos, por sus comentarios y sugerencias, que contribuyeron a mejorar la versión final del manuscrito. Conflictos de intereses: El manuscrito fue preparado y revisado con la participación de todos los autores, quienes declaramos que no existe ningún conflicto de intereses que ponga en riesgo la validez de los resultados presentados. Financiación: Este estudio fue financiado por la Fundación para la Promoción de la Investigación y la Tecnología del Banco de la República y La Universidad Tecnológica del Chocó 


\section{BIBLIOGRAFÍA}

1. ALBA-TERCEDOR, J. 1996. Macroinvertebrados acuáticos y calidad de las aguas de los ríos. IV Simposio del agua en Andalucía (SIAGA). Almería, Vol. II. p.203-213.

2. ALONSO, A. 2006. Valoración del efecto de la degradación ambiental sobre los macroinvertebrados bentónicos en la cabecera del río Henares. Ecosistemas, Asociación Española de Ecología Terrestre, 15(2):1-5.

3. APHA, AWWA, WEF. 2012. Standard Methods for examination of water and wastewater. 22nd ed. Washington: American Public Health Association; 1360p.

4. ARANGO, M.C.; ÁLVAREZ, L.F.; ARANGO, G.A.; TORRES, O.E.; DE J. MONSALVE, A. 2008. Calidad del agua de las quebradas La Cristalina y La Risaralda, San Luis, Antioquia. Revista EIA. 9:121-141.

5. ARMITAGE, P.D.; MOSS, D.; WRIGHT, J.F.; FURSE, M.T. 1983. The performance of a new biological water quality score based on macroinvertebrates over a wide range of unpolluted running-water sites. Wat. Res. 17:333-347.

6. ARMITAGE, P.D.; PETTS, G.E. 1992. Biotic score and prediction to assess the effects of water abstractions on river macroinvertebrates for conservation purposes. Aquatic Conservation: Marine and Freshwater Ecosystems. 2(1):1-17.

7. ASPRILLA, S.; MOSQUERA, Z.; RIVAS, M. 2006. Macroinvertebrados acuáticos como indicadores de calidad ecológica del agua en la parte media del río Cabí (Quibdó - Chocó). Rev. Asoc. Colom. Cien. Biol. 18:43-50.

8. BUSTAMANTE-TORO, C.A.; MARÍN-VILLEGAS, N.C.; CORREDOR-COY, N.V. 2011. Estudio de calidad ambiental en la quebrada La Florida, unidad de manejo de cuenca del río Quindío. Armenia - Quindío, Colombia. Rev. Asoc. Col. Cienc. (Col.), 23: 65-76.

9. CÁRDENAS, A.Y.; BISMARK, R.; LÓPEZ, M.; WOO, A; RAMÍREZ, E.; IBRAHIM, M. 2007. Biodiversidad de macroinvertebrados acuáticos y la calidad del agua en la subcuenca de los ríos Bul Bul y Paiwas, Matiguás, Nicaragua. Encuentro. 77:83-93.

10. CHARÁ, J.; PEDRAZA, G.; GIRALDO, L.; HINCAPIÉ, D. 2007. Efecto de los corredores ribereños sobre el es- tado de quebradas en la zona ganadera del río La Vieja, Colombia. Agrofor. Américas. 45:72-78.

11. CHARÁ, J. 2003. Manual para la evaluación biológica de ambientes acuáticos en microcuencas ganaderas. Fundación Centro para la Investigación en Sistemas Sostenibles de Producción Agropecuaria. CIPAV. Colombia. 52p.

12. CONTRERAS-MARTÍNEZ, E. 2013. Diversidad de Entomofauna acuática en tres ríos de la Ecoregión Darién, Choco biogeográfico (Colombia). Dugesiana. 20(2):243-250.

13. CÓRDOBA, K.E.; CASAS, L.; MOSQUERA-MURILLO, Z.; ASPRILLA, S. 2007. Composición y variación temporal del orden Ephemeroptera (Insecta) en los ríos Tutunendo y Catugadó, Quibdó (Chocó - Colombia). Rev. Asoc. Colom. Cienc. Biolo. 19:34-41.

14. DOMÍNGUEZ, E.; FERNÁNDEZ, H. 2009. Macroinvertebrados bentónicos sudamericanos. Sistemática y Biología. Fundación Miguel Lillo. Tucumán, Argentina. 654p.

15. DOMÍNGUEZ, E.; MOLINERI, C.; PESCADOR, M.L.; HUBBARD, M.D.; NIETO, C. 2006. Ephemeroptera of South América. In: Adís, J., Arias, JR., Reda-delgado, G. \& K.M Wantzen (Eds): Aquatic Biodiversity in Latín América (ABLA), Vol. 2 pensonft, sofia-moscow, 646p.

16. FIGUEROA, R.; VALDOVINOS, C.; ARAYA, E.; PARRA, O. 2003. Macroinvertebrados bentónicos como indicadores de calidad de agua del sur de Chile. Rev. Chilena Historia Natural. 76:275-285.

17. GARCÍA-MONTOYA, J.; CARMONA-BEDOYA, J.C.; MONTOYA-MORENO, Y. 2012. Caracterización de la calidad del agua de la parte alta de la microcuenca de la quebrada La Cimarronas, El Carmen de Viboral (Antioquia), utilizando macroinvertebrados acuáticos. Investigación, Biodiversidad y Desarrollo. 31(1):5-18.

18. GONZÁLEZ-CÓRDOBA, M.; ZÚÑIIGA, M.DELC.; MOSQUERA-MURILLO, Z.; SÁNCHEZ-VÁSQUEZ, S. 2016. Riqueza y distribución de Elmidae (Insecta: Coleoptera: Byrrhoidea) en el departamento del Chocó, Colombia. Revista Intropica. 11:85-95.

19. HAMMER, O.; HARPER, D.; RYAN, P.D. 2001. Past: Paleontological Statistics Software Package for Edu- 
cation and Data Analysis. Palaeontologia Electronica 4. Disponible en: http://palaeoelectronica. Org /2001_1/past/issue1_01.htm.

20. HOLDRIDGE, R.L. 1996. Ecología basada en zonas de vida. Instituto Inter. Americano de Cooperación para la Agricultura (IICA). San José de Costa Rica. p.1-6.

21. HOLZENTHAL. R.W.; BLAHNIK, R.J.; PRATHER, A.L.; KJER, K.M. 2007. Order Trichoptera Kirby, 1813 (Insecta), caddisflies. Zootaxa. 1668:639-698.

22. LARA-LARA, J.R.; ARREOLA, J.A.; CALDERÓN, L.E.; CAMACHO, V.F.; ESPINO, G.L.; ESCOFET, A.M.; ESPEJEL, M.; GUZMÁN, M.; LADAH, L.B.; LÓPEZ M.; MELING E.A.; MORENO P.; REYES H.; RÍOS E.; ZERTUCHE, J.A. 2008. Los ecosistemas costeros, insulares y epicontinentales, en Capital natural de México, vol. I: Conocimiento actual de la biodiversidad. Conabio, México, 109-134p.

23. MADERA, L.; ANGULO, L.C.; DÍAZ, L.C.; ROJANO, R. 2016. Evaluación de la calidad del agua en algunos puntos afluentes del río cesar (Colombia) utilizando macroinvertebrados acuáticos como bioindicadores de contaminación. Inf. Tecnológica. 27(4):103-110.

24. MARGALEF, R. 1983. Limnología. Editorial Omega. 1010p.

25. MERRITT, R.W.; CUMMINS, K.W. 1996. An Introduction to the Aquatic Insects of North America. 3 ed. Kendall /Hunt Publishing, Iowa, 862p.

26. MERRITT, R.W.; CUMMINS, K.W.; BERG, M.B. 2008. An introduction to the aquatic insects of North America. Dubuque, Kendall/Hunt Publishing Company. $1214 p$.

27. MEZA, A.M.; RUBIO, J.; DÍAS, L.; WALTEROS, J. 2012. Calidad de agua y composición de Macroinvertebrados acuáticos en la Subcuenca alta del río Chinchiná. Caldasia. 34(2):443-456.

28. MOSQUERA, Z.; BEJARANO, D.; ASPRILLA, S. 2006. Estudio del orden Trichoptera (Insecta) en dos ecosistemas lóticos del municipio de Quibdó, Chocó - Colombia. Libro de la Asociación Colombiana de Limnología. 1:85-91.

29. MOSQUERA, Z.; CÓRDOBA, K.E. 2015. Caracterización de la entomofauna acuática en cuatro quebradas de la cuenca del río San Juan, Chocó, Colombia. Rev. Acad. Colomb. Cienc. Ex. Fis. Nat. 39(150):67-76.
30. MOSQUERA, Z.; CÓRDOBA, K.E. 2017. Primer registro del genero Tikuna Savage, Flowers \& Porras (Ephemeroptera: Leptophlebiidae) para el Chocó, Colombia. En prensa.

31. NIEVES, G.; ROSAS, E.; HORNEDO, M. 2010. Biodiversidad de insectos acuáticos asociados a la cuenca del río Grande de Manatí. Departamento de recursos naturales y ambientales. Puerto Rico. 35p.

32. OCON, C.S.; RODRIGUEZ, A. 2004. Presence and abundance of Ephemeroptera and other sensitive macroinvertebrates in relation with habitat conditions in Pampean streams (Buenos Aires, Argentina). Arch. Hydrobiol. 159:473-487.

33. PRAT, N.; RIOS, B.; ACOSTA, R.; RIERADEVALL, $M$. 2009. Los macroinvertebrados acuáticos como indicadores de la calidad de las aguas. En: Domínguez, E.; Fernández, H, (eds), Macroinvertebrados bentónicos sudamericanos. Primera edición: p.631-654. San Miguel de Tucumán: Fundación Miguel Lillo.

34. RAMÍREZ, D.F.; TALERO, G.M.; LÓPEZ, R.H. 2013. Macroinvertebrados bentónicos y calidad del agua en un tramo del río Bogotá. Rev. U.D.C.A Act. \& Div. Cient. 16(1):205-214.

35. RESH, V.H. 2008. Which group is best? Attributes of different biologic assemblages used in freshwater biomonitoring programs. Environ. Monit. Assess. 138:131-138.

36. RICHARDSON, J.S.; MOORE, R.D. 2010. Stream and riparian ecology. (Chapter 13). In: Pike, R.G.; Redding, T.E.; Moore, R.D.; Winkler, R.D. Bladon, K.D. (eds). Compendium of Forest Hydrology and Geomorphology in British Columbia. B.C. Ministry of Forests and Range Res. Branch, Victoria, B.C. and FORREX Forest Res. Extension Partnership, Kamloops, B.C. Land Management Handbook (TBD).

37. RIVAS, M.; MOSQUERA, Z.; ASPRILLA, S. 2003. Calidad ecológica de las a guas de la parte media del río Cabí utilizando los macroinvertebrados como indicadores. Rev. Inst. Univ. Tecnológica del Chocó. 19:5963.

38. ROLDÁN, G.A. 2003. Bioindicación de la calidad del agua en Colombia: Uso del método BMWP/Col. Universidad de Antioquia. Colombia. 170p.

39. ROLDÁN, G.; ZÚÑIGA, M.DELC.; ZAMORA, H.; REINOSO, G.; ÁLVAREZ, L.F.; LONGO, M. 2014. Colombia. 
En: Alonso EguíaLis, P., Mora, J.M., Campbell, B., Springer, M., (Eds). Diversidad, conservación y uso de los macroinvertebrados dulceacuícolas de México, Centroamérica, Colombia, Cuba y Puerto Rico. Instituto Mexicano de Tecnología del Agua y Univ. Autónoma de Querétano, México y Universidad de Costa Rica, San José-Costa Rica.

40. ROLDÁN, G. 2016. Los macroinvertebrados como bioindicadores de la calidad del agua: cuatro décadas de desarrollo en Colombia y Latinoamérica. Rev. Acad. Colomb. Cienc. Ex. Fis. Nat. 40(155):254-274.

41. ROZO, A.M.; SALINAS, L.G. 2016. Inventario del orden Ephemeroptera (Insecta) en la quebrada Coquital, Serranía de Tripogadí, Departamento del Chocó, Colombia. Entomotropica. 31(1):1-13.

42. SALAS, Y.; GEOVO, S.; ASPRILLA, S. 2011. Caracterización de las comunidades perifíticas y de macroinvertebrados acuáticos presentes en el río Pacurita, corregimiento de Pacurita, Quibdó-Chocó-Colombia. Rev. Biodiversidad Neotropical. 1(2):98-104.

43. SÁNCHEZ, M.; LEÓN, C.W.; ROJAS, J.; VARGAS, R. 2006. Calidad de agua, invertebrados y bioindicación en el río Magdalena en el norte del departamento del Huila. Pág. 92 en: F.A. Villa, C. A. Rivera, G.R. Flórez \& M. Avellaneda (Eds), Resúmenes VII seminario Colombiano de Limnología. Universidad del Tolima-ACL-Limnos. 2006.

44. SEGNINI, S. 2003. El uso de los macroinvertebrados bentónicos como indicadores de la condición ecológica de los cuerpos de agua corriente. Ecotropico. 16(2):45-63.

45. SHIEH, S.H.; YANG, P.S. 2000. Community structure and functional organization of aquatic insects in an agricultural mountain stream of Taiwan: 1985-1986 and 1995-1996. Zool Stud. 39:191-202.

46. SPRINGER, M.; RAMÍREZ, A.; HANSON, P. 2010. Macroinvertebrados de agua dulce de Costa Rica I. Rev. Biol. Trop. 58 (Suppl. 4):97-136.
47. SPRINGER, M. 2010. Biomonitoreo. En: Springer, M., Ramírez A. \& P. Hanson (eds.). Macroinvertebrados de agua dulce de Costa Rica I. Rev. Biol. Trop. 58 (Suppl. 4): 53-59.

48. TORRES, Y.; ROLDÁN, G; ASPRILLA, S.; RIVAS, T. 2006. Estudio preliminar de algunos aspectos ambientales y ecológicos de las comunidades de peces y macroinvertebrados acuáticos en el río Tutunendo, Chocó-Colombia. Rev. Acad. Colomb. Cienc. Ex. Fis. Nat. 114(30):67-76.

49. TRIGAL-DOMÍNGUEZ, C.; FRENÁNDEZ-ALÁEZ, C.; GARCÍA- CRIADO, F. 2009. Ecological assessment of highly heterogeneous systems: The importance of taxonomic sufficiency. Limnologica 4:208-214.

50. VÁSQUEZ-RAMOS, J.M.; REINOSO, G. 2012. Estructura de la fauna béntica en corrientes de los Andes colombianos. Rev. Col. Entomología. 38(2):351-358.

51. ZAMORA, H. 2015. Macroinvertebrados acuáticos registrados durante la época de lluvias en tres ríos del piedemonte llanero de Colombia. Rev. Colombiana Cienc. Anim. 7(2):139-147.

52. ZÚÑIGA, M.DELC.; CHARÁ, J.C.; GIRALDO, L.P.; CHARÁ-SERNA, A.M.; PEDRAZA, G.X. 2013. Composición de la comunidad de macroinvertebrados acuáticos en pequeñas quebradas de la región andina colombiana, con énfasis en la entomofauna. Dugesiana 20(2):263-277.

53. ZÚĨIIGA, M.C.; CARDONA, W.; MOLINERI, C.; MENDIVIL, J.; CULTID, C.; CHARÁ, A.M.; GIRALDO, A. 2014. Entomofauna acuática del Parque Nacional Natural Gorgona, Pacífico Colombiano, con énfasis en Ephemeroptera y Plecoptera. Rev. Biol. Trop. 62(Suppl. 1):221-241.

Recibido: Noviembre 26 de 2016

Aceptado: Mayo 11 de 2017

Cómo citar:

Mosquera Murillo, Z.; Mosquera Mosquera, M.M. 2017. Diversidad de la entomofauna acuática y calidad de agua en quebradas del rio San Juan, Chocó - Colombia. Rev. U.D.C.A Act. \& Div. Cient. 20(1): 149-161. 